\title{
Identifying the Risk Factor and Prevention of Limb Ischemia in Extracorporeal Membrane Oxygenation with Femoral Artery Cannulation
}

\author{
Chih-Chien Yen, MD, ${ }^{1,2}$ Chih-Hong Kao, ${ }^{1}$ Chien-Sung Tsai, PhD,${ }^{1,3}$ Shin-Han Tsai, $\mathrm{PhD}^{2}$ \\ ${ }^{1}$ Division of Cardiovascular Surgery, Department of Surgery, Taoyuan Armed Forces General Hospital, Taoyuan, Taiwan; ${ }^{2}$ Taipei \\ Medical University Graduate Institute of Injury Prevention and Control; ${ }^{3}$ Department of Cardiovascular Surgery, Tri-Service \\ General Hospital, National Defense Medical Center, Taipei, Taiwan
}

\section{ABSTRACT}

Objective: Application of extracorporeal membrane oxygenation (ECMO) for life support has been widely used in various fields of resuscitation. When the common femoral artery (CFA) is used during cannulation for ECMO support in adults, it is often complicated by limb ischemia. Placement of distal perfusion catheter (DPC) can reduce the incidence of limb ischemia and increases the likelihood of limb preservation, but selection criteria is uncertain.

Methods: This is a retrospective study. Data was reviewed for patients in one medical center who were supported by venoarterial extracorporeal membrane oxygenation (VAECMO) via CFA cannulation percutaneously between January 2008 and June 2014. Two groups were divided into noischemia and ischemic limb. Age, sex, height, weight, body surface area (BSA), cannula size, femoral artery diameter, comorbidity, acute physiology and chronic health evaluation (APACHE) II score, vasoactive-inotropic score (VIS) and mortality rate were analyzed. Doppler was used by measuring the distal pulsation in the dorsalis pedis and posterior tibial artery to select the patients. A DPC was prophylactically inserted percutaneously into the superficial femoral artery for antegrade flow to the extremity in the patients who met selection criteria.

Results: 139 (43.6\%) patients were included in the study and limb ischemia occurred in $46(33 \%)$ of 139 . There was a significant difference between the no-ischemia group and the ischemia group in age $(55.5 \pm 14.2$ versus $63.2 \pm 13.2$; $P<.001)$, common femoral artery diameter $(0.82 \pm 0.14$ versus $0.63 \pm 0.17 ; P<.001)$, known peripheral artery occlusive disease $(9 \%$ versus $24 \% ; P<.001)$ and VIS $(12.1 \pm 8.1$ versus $15.8 \pm 10.1 ; P<.001)$. Mortality rate was higher in the ischemia group $(46 \%$ versus $26 \% ; P<.001) .11$ patients who met the selection criteria had a DPC prophylactically inserted and no ischemia limb occurred.

Conclusion: Smaller common femoral artery diameter $(\leq 6.3 \mathrm{~cm})$; known peripheral arterial occlusive disease; higher

Received April 22, 2017; accepted May 6, 2017.

Correspondence: Chib-Chien Yen, MD, Division of Cardiovascular Surgery, Department of Surgery, Taoyuan Armed Forces General Hospital, 168, Chung Hsing Rd., Taoyuan City, 32551 Taiwan, Republic of China; +886 347-99595 (e-mail: cvsyen@gmail.com).
VIS ( $\geq 15.8$ ); absence of distal pulsation pre-cannulation or immediately after post-cannulation or 4 hrs later have higher risk of limb ischemia when CFA cannulation is used for VAECMO. Due to this, the mortality and morbidity rate increases when limb ischemia occurs. A DPC should be prophylactically inserted in high-risk patients who meet selection criteria.

\section{INTRODUCTION}

In intensive care medicine, extracorporeal membrane oxygenation (ECMO) is a treatment that uses a pump to circulate blood through an artificial lung back into the bloodstream of a very ill patient. This system provides heart-lung bypass support outside of the patient's body. There are two

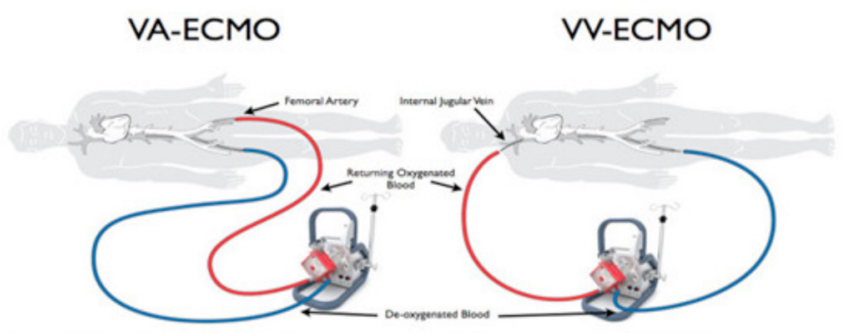

Figure 1. Two types of ECMO: venovenous (VV) and venoarterial (VA).

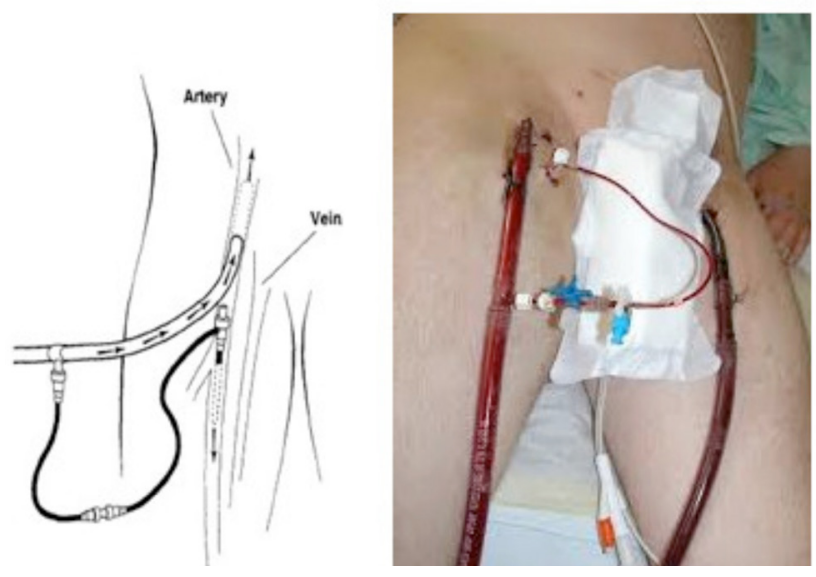

Figure 2. A DPC was inserted percutaneously into the superficial femoral artery for antegrade flow to the extremity. 


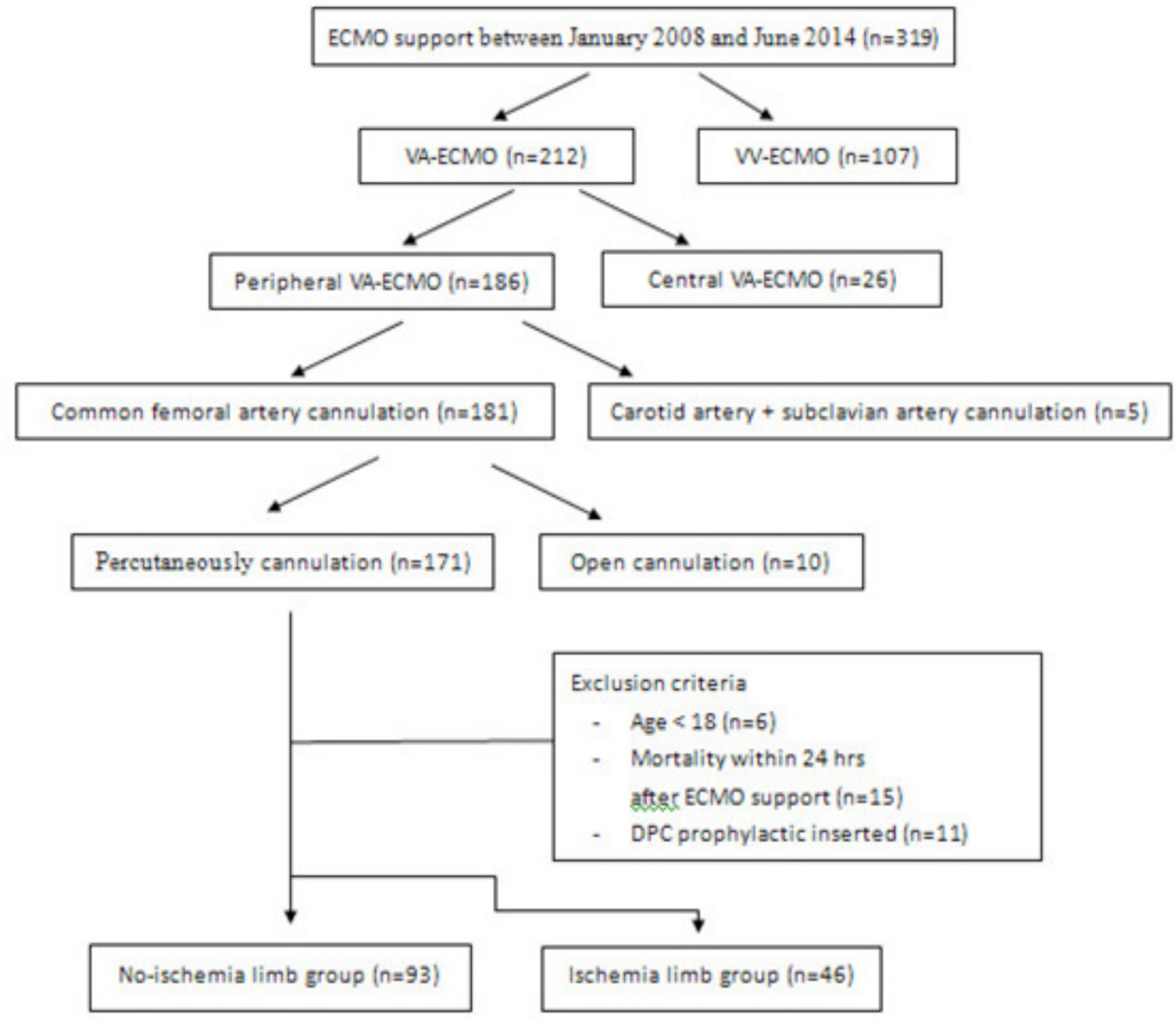

Figure 3. Flowchart of patient selection and outcomes.

types of ECMO: venovenous (VV) and venoarterial (VA). In VV-ECMO, the blood is returned to the venous system and in VA-ECMO the blood is returned to the arterial system (Figure 1). Both provide lung support, but only VA-ECMO provides heart support.

ECMO has resulted in improvements in both survival and quality of life for an increasing number of patients with heart and lung failure. Despite increasing experience with ECMO and recent technical improvements, the mortality of patients receiving ECMO remains high. In-hospital survival rate of patients with VA-ECMO varies from $30 \%$ to $50 \%$ according to the cause of the cardiac dysfunction [Elsharkawy 2010]. Outcome of patients on ECMO is influenced not only by factors independent of ECMO (patient illness severity, type of illness, other organ support) but also by the potential complications related to ECMO. Common complications of VA-ECMO included thrombosis (1-22\%), bleeding (5-79\%), infection (17-49\%), and limb ischemia (13-25\%) [Mehta 2015].

A cannula is usually placed percutaneously by the Seldinger technique. In VA-ECMO, a venous cannula is usually placed in the common femoral vein for extraction and an arterial cannula is usually placed into the common femoral artery for infusion because the insertion is simpler and more rapid [Madershahian 2006]. When the common femoral artery is used during cannulation for ECMO support in adults it is often complicated by limb ischemia due to femoral artery obstruction. 


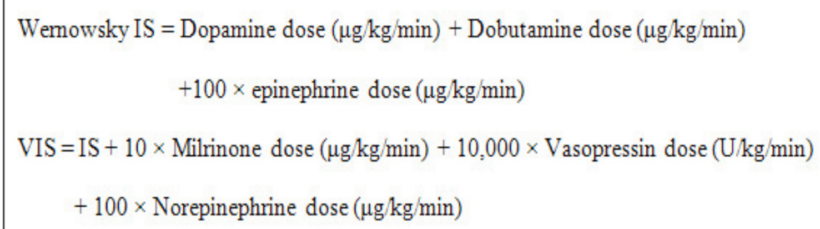

Figure 4. Vasoactive-inotropic score (VIS).

According to the 2007 Inter-Society Consensus for the Management of Peripheral Arterial Disease (TASC II), acute limb ischemia is defined as a sudden decrease in limb perfusion that causes a potential threat to limb viability (manifested by ischemic rest pain, ischemic ulcers, and/or gangrene) [Norgren 2007]. A delay in initiation of therapy may lead to an irreversibly injured limb. Amputation occurs in about $20-30 \%$ of all limb ischemia patients and $50 \%$ mortality in those that require amputation.

Distal perfusion catheter (DPC) (Figure 2) can be done either via a surgical end-to-side graft from the ECMO circuit into the superficial femoral artery, or through a catheterbased insertion of a reperfusion cannula via retrograde insertion from distal limb vessels. The use of a DPC to perfuse distal to the entry site of ECMO cannulas can reduce the incidence of limb ischemia and increase the likelihood of limb preservation, but selection criteria is uncertain.

This study describes the experience of a single medical center. The aim of this study was to examine pre-cannulation variables that were associated with limb ischemia and identify selection criteria for using DPC for prevention of limb ischemia.

\section{METHODS}

This study was conducted at Tri-Service General Hospital, a 1850-bed academic teaching hospital affiliated with the National Defense Medical Center in Taipei, Taiwan. Data were obtained from retrospective review of patient medical records.

This retrospective study involved 139 patients who were

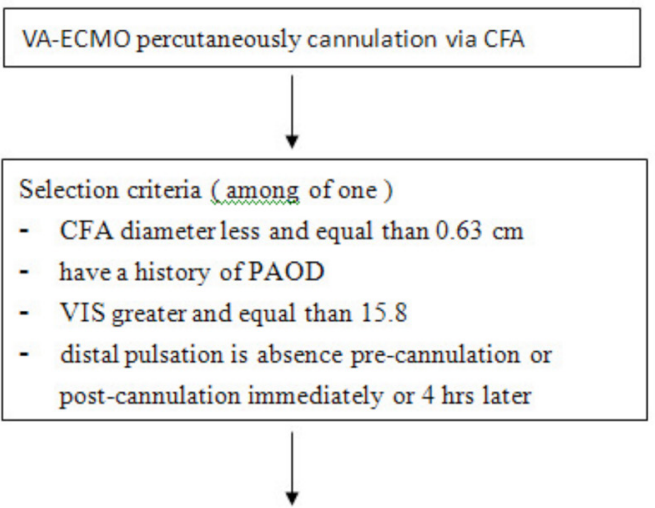

Use a standard 6F Arrow-Flex cordis catheter is placed percutaneously

Figure 5. Treatment protocol.
Patient and ECMO-Related Variables for Developing Limb Ischemia

\begin{tabular}{lccc}
\hline & No ischemia $(\mathrm{n}=93)$ & Ischemia $(\mathrm{n}=46)$ & $P$ \\
\hline Age, $y$ & $55.5 \pm 14.2$ & $63.2 \pm 13.2$ & $<.001$ \\
Male sex, $\mathrm{n}(\%)$ & $41(44)$ & $18(40)$ & .51 \\
Body height, $\mathrm{cm}$ & $169.8 \pm 5.6$ & $167.2 \pm 5.8$ & .85 \\
Body weight, kg & $61.3 \pm 8.7$ & $64.8 \pm 8.2$ & .27 \\
BSA, m ${ }^{2}$ & $1.64 \pm 0.07$ & $1.66 \pm 0.06$ & .93 \\
Cannula size & $16.5 \pm 0.8$ & $16.6 \pm 0.8$ & .71 \\
CFA diameter & $0.82 \pm 0.14$ & $0.63 \pm 0.17$ & $<.001$ \\
Co-morbidity & & & \\
Diabetes mellitus, $\mathrm{n}(\%)$ & $16(17)$ & $10(22)$ & .621 \\
Hypertension, $\mathrm{n}(\%)$ & $28(30)$ & $17(37)$ & .55 \\
Uremia, $\mathrm{n}(\%)$ & $10(11)$ & $8(17)$ & .55 \\
PAOD, $\mathrm{n}(\%)$ & $8(9)$ & $11(24)$ & $<.001$ \\
APACH II score & $18.9 \pm 14.7$ & $19.6 \pm 16.4$ & .76 \\
VIS & $12.1 \pm 8.1$ & $15.8 \pm 10.1$ & $<.001$ \\
Mortality, $\mathrm{n}(\%)$ & $24(26)$ & $21(46)$ & $<.001$ \\
& & &
\end{tabular}

supported by VA-ECMO via CFA cannulation percutaneously between January 2008 and June 2014. Exclusion criteria were: patient age less than 18 , mortality within 24 hours after VA-ECMO support, and DPC use for prevention of ischemia limb. Limb ischemia was the primary variable (Figure 3).

The ischemia group was defined as the patients requiring an intervention because of the development of lower extremity ischemia. The patients in the no-ischemia group did not develop significant ischemia. Variables selected for analysis between the 2 groups were age, sex, height, weight, body surface area (BSA), cannula size, femoral artery diameter, comorbidities and mortality rate. In addition, we calculated the VIS [Gaies 2014] (Figure 4) and APACHE II score for each patient immediately before VA-ECMO cannulation.

Doppler was used by measuring the distal pulsation in the dorsalis pedis artery and posterior tibial artery to select the patients at risk. After ECMO was established, a standard 6F Arrow-Flex cordis catheter was placed percutaneously distal to the arterial cannula into the SFA and connected to the side port of the arterial line to provide antegrade femoral blood flow in the patient who had an absence of distal pulsation pre-cannulation or postcannulation. Heparin was used for anticoagulation and maintaining the target activated clotting time (ACT) between 160-180s.

\section{Statistical Analysis}

Categorical variables were compared between groups with Fisher exact test and continuous variables were presented as mean \pm standard deviation and compared using Mann-Whitney $\mathrm{U}$ test. A $P$ value $<.05$ was considered to be statistically significance. Data analyses were performed using the Statistical Package for the Social Sciences, version 16.0 (SPSS, Chicago, IL, USA). 


\section{RESULTS}

Between January 2008 and June 2014, 319 patients underwent ECMO support. 171 patients were cannulated percutaneously via the common femoral artery for VA-ECMO. 11 patients who had DPC prophylactically inserted to prevent limb ischemia were excluded. 139 (43.6\%) patients were included in the study and limb ischemia occurred in 46 (33\%) of 139 (Figure 1).

In comparing the 2 groups (no-ischemia versus ischemia), some clinical variables were predictive of the development of limb ischemia. There was no significant difference in sex, weight, height, BSA $(1.64 \pm 0.07$ versus $1.66 \pm 0.06 ; P=.93)$ and cannula size $(16.5 \pm 0.8$ versus $16.6 \pm 0.8 ; \mathrm{P}=.73)$ between the 2 groups. There was a significant difference between the no-ischemia group and the ischemia group in age (55.5 \pm 14.2 versus $63.2 \pm 13.2 ; P<.001)$ and CFA diameter $(0.82 \pm 0.14$ versus $0.63 \pm 0.17 ; P<.001)$. History of diabetes mellitus (17\% versus $22 \% ; P=.621$ ), hypertension $(30 \%$ versus $37 \%$; $P=.55)$ and uremia $(11 \%$ versus $17 \% ; P=.29)$ showed no significant difference between groups, but known peripheral artery occlusive disease (PAOD) $(9 \%$ versus $24 \%$; $P<.001)$ showed significant difference.

We used the APACHE II score to assess the severity of each patient before cannulation; there was no significant difference between the 2 groups $(18.9 \pm 14.7$ versus $19.6 \pm 16.4 ; P=.76)$. To assess the contribution of vasopressors to ischemia, we calculated the VIS of each patient before cannulation. There was a significant difference between the 2 groups $(12.1 \pm 8.1$ versus $15.8 \pm 10.1 ; P<.001)$. Mortality rate was higher in the ischemia group ( $46 \%$ versus $26 \% ; P<.001)$ (Table).

Doppler was used by measuring the distal pulsation in the dorsalis pedis artery and posterior tibial artery. 11 patients who met selection criteria had DPC prophylactic inserted and no ischemia limb occurred.

\section{DISCUSSION}

Extracorporeal Life Support Organization Registry data confirm that the number of patients being supported by ECMO is increasing [ELSO 2010]. Most patients undergo percutaneous cannulation via CFA due to the procedure being simpler and more rapid [Madershahian 2006]. In our study, 181 patients were cannulated via the common femoral artery for VA-ECMO. Of these, 171 (94\%) underwent cannulation percutaneously.

When the femoral artery is used during cannulation for VA-ECMO support in adults, distal limb ischemia is a welldocumented complication and the incidence rate is $20-50 \%$ [Mehta 2015; Madershahian 2006; Greason 1995; Foley 2010; Gander 2010]. Our study demonstrated clinically significant arterial compromise leading to limb ischemia in 46 patients $(30 \%)$.

In predictive variables of limb ischemia compared with other studies, Gander et al demonstrated that there is no significant differences in age, sex, BSA, and cannula size in the pediatric population [Gander 2010]. Foley et al showed that younger patients may be at increased risk for limb ischemia due to smaller femoral artery diameter and have less collateralization of the lower extremity arterial circulation, but the case number totalled only 43 patients [Foley 2010]. Gaies et al demonstrated in pediatric cardiac surgery experience that VIS was strongly and significantly associated with morbidity and mortality [Gaies 2014]. Our data found that the ischemia group had smaller CFA diameter and older patient age. We also found that known peripheral arterial occlusive disease and higher VIS contribute to a higher risk for limb ischemia. This may be due to older patients having more incidence of poor peripheral vessel condition and higher VIS that can reduce peripheral arterial circulation. In our study, smaller common femoral artery diameter, known peripheral arterial occlusive disease, and higher VIS were predictive of the development of limb ischemia.

When limb ischemia occurs, mortality and morbidity rates also increase. Finding methods to prevent limb ischemia is very important. In some studies, a DPC is suggested to decrease rates of ischemia but selection criteria is uncertain [Madershahian 2006; Foley 2010; Huang 2004]. It is not necessary to place DPC in every patient. When clinical signs of ischemia arise, a DPC is placed for increased distal perfusion but compartment syndrome might occur after reperfusion starts [Huang 2004]. Some protocols have been established to address these limb complications. Huang et al measured the mean arterial pressure of the superficial femoral artery, suggesting that a distal mean pressure less than $50 \mathrm{mmHg}$ is an appropriate criterion for placing a DPC. But this is an invasive procedure [Huang 2004]. Schachner et al used nearinfrared spectroscopy (NIRS) for transcutaneous measurement of tissue oxygen saturation to evaluate ischemia but the sensitivity is low [Schachner 2008].

We aim to identify criteria to select high-risk patients for insertion of a DPC. According to our study, risk factors were defined as (1) CFA diameter less than or equal to $0.63 \mathrm{~cm}$; (2) a history of PAOD; and (3) VIS greater than or equal to 15.8 . We also used duplex ultrasonography to detect the dorsalis pedis and posterior tibial artery pulsation pre-cannulation, immediately after post-cannulation and 4 hours later. Our protocol stated that if the patient had any risk factors or if the distal pulsation was absent pre-cannulation, immediately after post-cannulation or 4 hours later, DPC was used (Figure 5). There was no limb ischemia occurrence in 11 patients with DPC prophylactically inserted.

\section{Study Limitations}

There were two limitations to our study. First, the study sample was small, though compared with recent studies, our case numbers are high. Second, our findings are based on a single-center study. Multi-center studies employing the same protocol in a larger number of patients will be required in the future.

\section{Conclusion}

In our study, smaller common femoral artery diameter, known peripheral arterial occlusive disease, higher VIS, and absence of distal pulsation pre-cannulation or post-cannulation have higher risk of limb ischemia with CFA cannulation 
for VA-ECMO. Mortality and morbidity rates then increased when limb ischemia occurred. A DPC should be prophylactically inserted in high-risk patients who meet selection criteria.

\section{REFERENCES}

Elsharkawy HA, Li L, Esa WA, et al. 2010. Outcome in patients who require venoarterial extracorporeal membrane oxygenation support after cardiac surgery. J Cardiothorac Vasc Anesth 24:946-51.

ELSO. ECMO Registry of the Extracorporeal Life Support Organization (ELSO) Mich; Ann Arbor: 2010.

Foley PJ, Morris RJ, Woo EY, et al. 2010. Limb ischemia during femoral cannulation for cardiopulmonary support. J Vasc Surg 52:850-3.

Gaies MG, Jeffries HE, Niebler RA, et al. 2014. Vasoactive-inotropic score is associated with outcome after infant cardiac surgery: an analysis from the pediatric cardiac critical care consortium and virtual PICU system registries. Pediatr Crit Care Med 15:529-37.

Gander JW, Fisher JC, Reichstein AR, et al. 2010. Limb ischemia after common femoral artery cannulation for venoarterial extracorporeal membrane oxygenation: an unresolved problem. J Pediatr Surg 45:2136-40.
Greason KL, Hemp JR, Maxwell JM, Fetter JE, Moreno-Cabral RJ. 1995. Prevention of distal limb ischemia during cardiopulmonary support via femoral cannulation. Ann Thorac Surg 60:209-10.

Huang SC, Yu HY, Ko WJ, Chen YS. 2004. Pressure criterion for placement of distal perfusion catheter to prevent limb ischemia during adult extracorporeal life support. J Thorac Cardiovasc Surg 128:776-7.

Madershahian N, Nagib R, Wipperman J, et al. 2006. A simple technique of distal limb perfusion during prolonged femoro-femoral cannulation. J Card Surg 21:168-9.

Mehta H, Eisen HJ, Cleveland JC, et al. Indications and complications for VA-ECMO for cardiac failure. American College of Cardiology Jul $14,2015$.

Norgren L, Hiatt WR, Dormandy JA, et al. 2007. Inter-Society Consensus for the Management of Peripheral Arterial Disease (TASC II). J Vasc Surg 45 SupplS:S5.

Schachner T, Bonaros N, Bonatti J, et al. 2008. Near infrared spectroscopy for controlling the quality of distal leg perfusion in remote access cardiopulmonary bypass. Eur J Cardiothorac Surg 34:1253-4. 